\title{
Leiomioma epitelioide en el párpado. Reporte de un caso
}

\section{Epithelioid leiomyoma of the eyelid. A case report}

\author{
Albanydia Montiel-Arista ${ }^{1 *}$, Alfredo Medina-Zarco ${ }^{2}$, Fernando E. de la Torre-Rendón ${ }^{3}$, \\ Claudia Herrera-Dorantes ${ }^{3}$ y Diana I. González-Hinojosa ${ }^{2}$
}

${ }^{1}$ Servicio de Oftalmología; ${ }^{2}$ Servicio de Órbita, Párpados y Vías Lagrimales; ${ }^{3}$ Servicio de Patología. Hospital Regional Lic. Adolfo López-Mateos, Instituto de Seguridad y Servicios Sociales de los Trabajadores (ISSSTE), Ciudad de México, México

\section{Resumen}

Se describe el caso clínico sobre un tumor de estirpe histológica poco frecuente en cuanto a su localización, en un paciente de sexo femenino de 18 años de edad, quien inició con un nódulo eritematoso y doloroso en el párpado superior derecho, que se trató de forma conservadora, con buen resultado terapéutico; sin embargo, posterior a 1 año de evolución recidivó en el mismo sitio, por lo que se realizó biopsia. El reporte histopatológico fue un leiomioma epitelioide. Los párpados pueden ser el sitio de desarrollo de tumores poco comunes, en la mayoría de los casos estromales. La conducta terapéutica ante un tumor palpebral exige biopsia y análisis histopatológico para establecer el diagnóstico y tratamiento correctos.

Palabras clave: Pseudotumor. Tumor palpebral. Leiomioma epitelioide. Células fusiformes. Actina específica a músculo. Biopsia excisional.

\section{Abstract}

We present the case of a rare histological eyelid tumor in an 18-year-old female with a painful erythematous nodule in her upper right eyelid for which conservative treatment was initiated; however, she suffered a relapse and then a biopsy was performed. Histopathology examination revealed an epithelioid leiomyoma. Rare tumors may develop in the eyelids, mainly of the stromal type. In cases of eyelid tumors, biopsy and histopathological examination are mandatory to confirm clinical diagnosis and treatment.

Key words: Pseudotumor. Eyelid tumor. Epithelioid leiomyoma. Spindle cells. Muscle-specific actin. Excisional biopsy.

\section{Introducción}

Las lesiones palpebrales tumoralesy pseudotumorales pueden tener el mismo aspecto clínico, por lo que la piedra angular en el diagnóstico y pronóstico de la entidad lo constituye su naturaleza histopatológica. El color uniforme, crecimiento limitado, contorno regular, conservación de las estructuras del borde palpebral, ausencia de induración y/o ulceración son características de las lesiones pseudotumorales, y los ejemplos más habituales son los nódulos y quistes benignos, como el orzuelo, chalazión, quiste de inclusión epidérmica, quiste epidermoide, dermoide, sebáceo, de Zeis, de Moll, hidrocistoma ecrino, acné miliar, comedones,

\section{Correspondencia:}

*Albanydia Montiel-Arista

Av. Universidad, 1321

Col. Florida, Del. Álvaro Obregón

Fecha de recepción: 26-11-2018

C.P. 01030, Ciudad de México, México

E-mail: dra.albanydia.uaeh@gmail.com
Fecha de aceptación: 09-09-2019

DOI: 10.24875/RMO.M19000096
Disponible en internet: 07-11-2019 Rev Mex Oftalmol. 2020;94(6):279-283

www.rmo.com.mx 0187-4519/@ 2019 Sociedad Mexicana de Oftalmología. Publicado por Permanyer México. Este es un artículo open access bajo la licencia CC BY-NC-ND (http://creativecommons.org/licenses/by-nc-nd/4.0/). 
Tabla 1. Lesiones palpebrales tumorales y pseudotumorales

\begin{tabular}{|c|c|}
\hline Lesiones benignas & Lesiones malignas \\
\hline $\begin{array}{l}\text { Papiloma epidermoide } \\
\text { Papiloma basocelular } \\
\text { Queratosis folicular invertida } \\
\text { Queratosis actínica } \\
\text { Verruga vulgar } \\
\text { Tricolemoma }\end{array}$ & $\begin{array}{l}\text { Carcinoma basocelular (más frecuente). } \\
\text { Carcinoma de células escamosas } \\
\text { Carcinoma sebáceo }\end{array}$ \\
\hline $\begin{array}{l}\text { Principales lesiones nodulares eritematosas de aspecto inflamatorio } \\
\text { Chalazión } \\
\text { Hemangioma capilar } \\
\text { Hiperplasia pseudocarcinomatosa } \\
\text { Granuloma piogénico } \\
\text { Nevo de células epitelioides o fusiformes } \\
\text { Adenoma pleomorfo } \\
\text { Acroespiroma exocrino } \\
\text { Pilomatricoma } \\
\text { Tumor glómico } \\
\text { Xantogranuloma juvenil } \\
\text { Hiperplasia endotelial intravascular benigna }\end{array}$ & $\begin{array}{l}\text { Carcinoma basocelular } \\
\text { Tumor de Merkel } \\
\text { Melanoma maligno nodular } \\
\text { Adenocarcinoma mucinoso } \\
\text { Angiosarcoma } \\
\text { Sarcoma de Kaposi }\end{array}$ \\
\hline $\begin{array}{l}\text { Principales lesiones nodulares sin modificación de coloración de los tegumentos o } \\
\text { pigmentados } \\
\text { Chalazión } \\
\text { Tumor celular granular } \\
\text { Nevo dérmico } \\
\text { Nevo de células epitelioides o fusiformes } \\
\text { Siringoma } \\
\text { Molusco contagioso } \\
\text { Nódulo pseudorreumatoide } \\
\text { Nódulo de sarcoidosis }\end{array}$ & $\begin{array}{l}\text { Carcinoma basocelular } \\
\text { Carcinoma sebáceo } \\
\text { Melanoma maligno nodular } \\
\text { Adenoma mucinoso }\end{array}$ \\
\hline $\begin{array}{l}\text { Principales lesiones nodulares ulceradas o con un cráter de queratina } \\
\text { Queratoacantoma } \\
\text { Orzuelo-chalazión } \\
\text { Acroespiroma exocrino } \\
\text { Tricoepielioma } \\
\text { Tricolemoma } \\
\text { Fascitis nodular }\end{array}$ & $\begin{array}{l}\text { Carcinoma basocelular } \\
\text { Carcinoma epidermoide }\end{array}$ \\
\hline
\end{tabular}

etc. ${ }^{1-3}$ (Tabla 1). Los párpados pueden ser el sitio de desarrollo de tumores poco comunes, en la mayoría de los casos estromales. Los tumores de músculo liso en los párpados, como los leiomiomas y los leiomiosarcomas, son muy raros ${ }^{4}$. Hui, et al., del Bascom Palmer Eye Institute, en 2010 realizaron el primer reporte de caso de leiomioma primario en el párpado inferior izquierdo, en un niño de 10 años, y se presentaba como un nódulo doloroso recurrente ${ }^{5}$.

Los leiomiomas son lesiones de lento crecimiento, originarias del músculo liso. Se ha descrito su localización a nivel intraorbitario en 25 casos, sus características histológicas y la ausencia de recurrencia tras la resección total apoyan su comportamiento benigno ${ }^{6}$. Por otro lado, Lin J, et $\mathrm{al}^{7}$. reportaron ocho casos de angioleiomioma, de los cuales seis ocurrieron intraconales y dos a nivel palpebral, y fueron más frecuentes en el sexo masculino. La conjuntiva palpebral es otro de los sitios poco comunes donde se ha reportado la presencia de leiomioma ${ }^{8}$.

\section{Presentación del caso}

Se trata de un paciente de sexo femenino, de 18 años de edad, residente de la Ciudad de México, sin antecedentes personales patológicos de importancia. Inicia su padecimiento con la aparición de un nódulo eritematoso y doloroso en el tercio interno del borde palpebral superior derecho, el cual fue tratado de manera conservadora con antibiótico y antiinflamatorio en ungüento oftálmico y evolucionó de manera favorable a la resolución. Sin embargo, posterior a 1 año de evolución, recidiva dicho tumor en el mismo sitio, involucrando el punto lagrimal (Figs. 1 y 2). Se decidió biopsia incisional del nódulo sin obtener resultados concluyentes, por lo que en un segundo tiempo se 


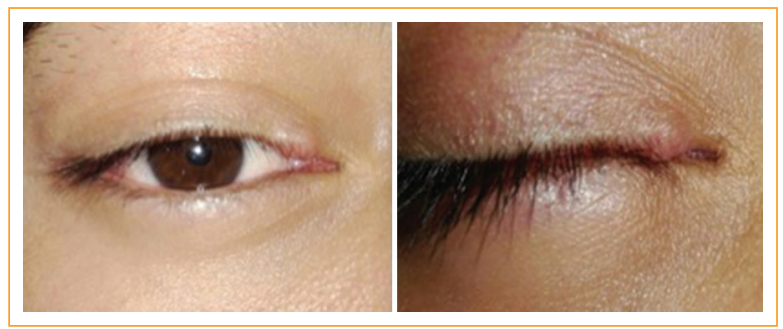

Figura 1. Aspecto clínico externo de tumoración en el tercio interno del párpado superior derecho.

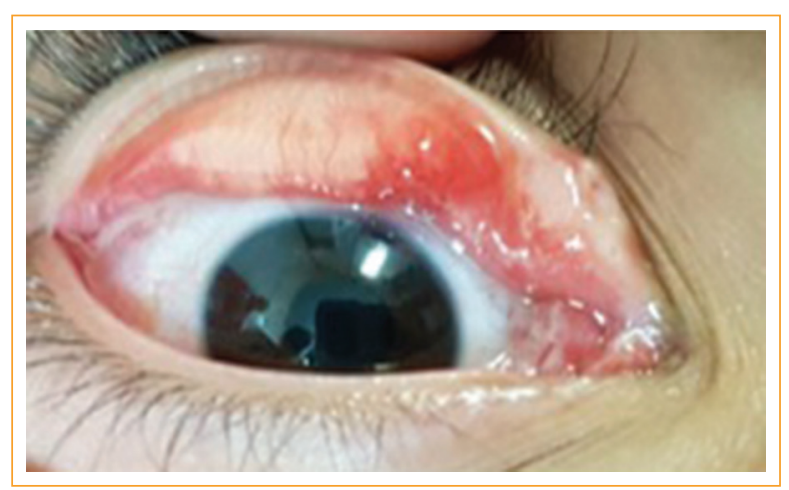

Figura 2. Aspecto clínico de tumoración en el tercio interno del párpado superior derecho.

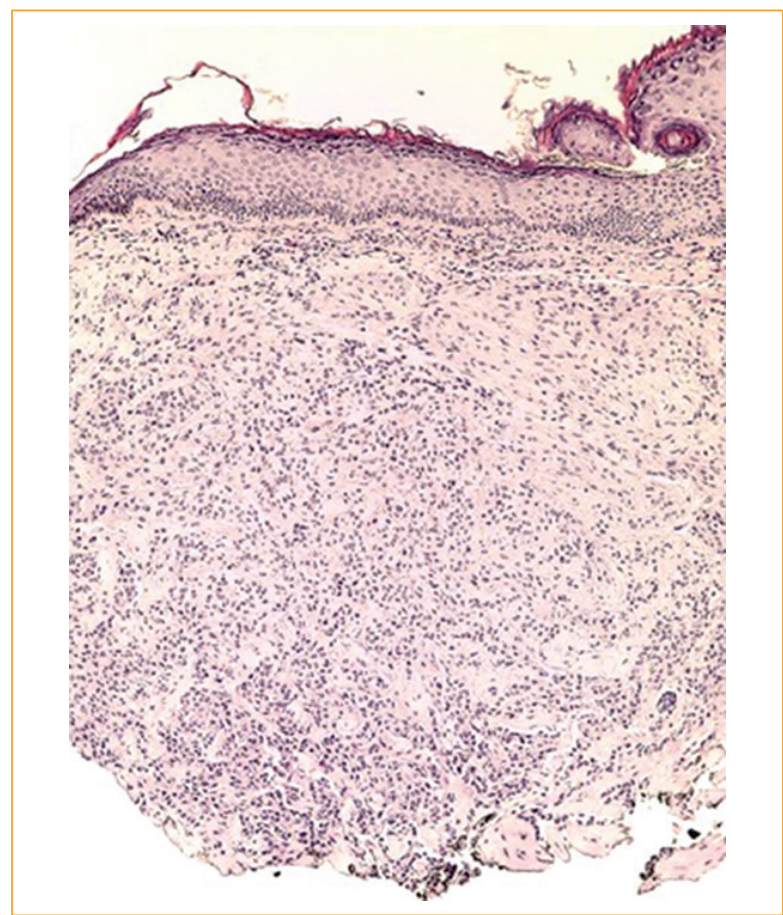

Figura 3. Piel de párpado que a nivel del estroma dérmico muestra proliferación celular de patrón acordonado, mal delimitado, que diseca las fibras colágenas (H\&E 100x).

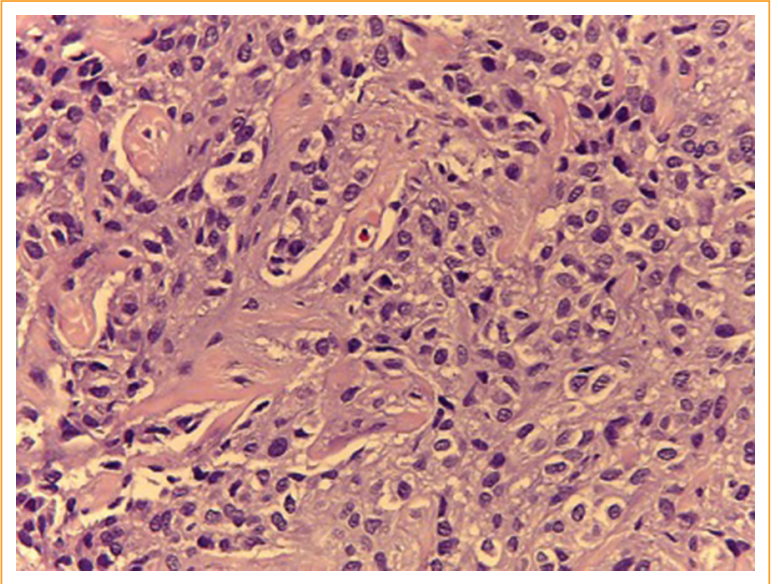

Figura 4. Proliferación compuesta por células

epitelioides, redondas, de citoplasma abundante pálido, que muestran leve variación en su forma y tamaño (H\&E $400 x)$.

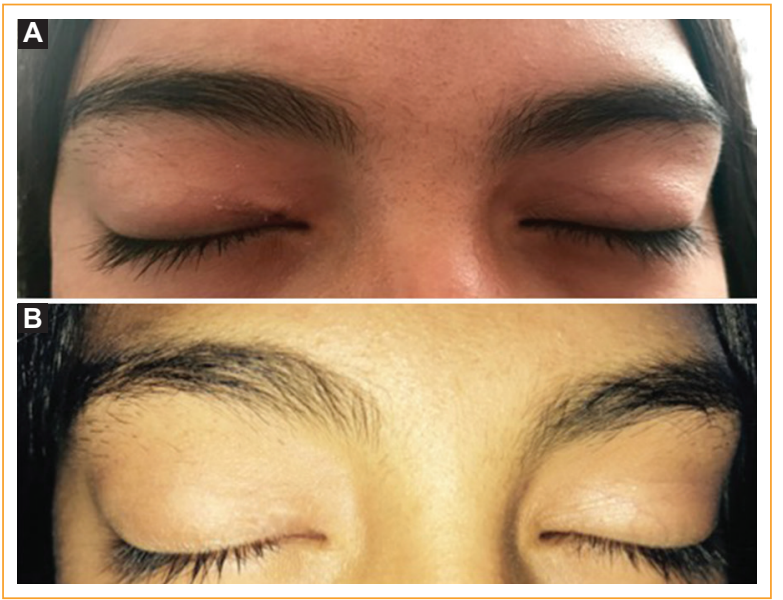

Figura 5. A: Aspecto clínico en las primeras semanas del postoperatorio B: Aspecto clínico después de 1 mes de postoperatorio.

realiza biopsia excisional para su interpretación histopatológica (Figs. 3 y 4) y reparación de la vía lagrimal, así como del párpado superior (Fig. 5).

Al estudio microscópico se encontró un tumor en el estroma de la piel palpebral, no encapsulado. Estaba formado por células fusiformes y epitelioides poco cohesivas y mezcladas con colágeno. No mostraron atipia y la actividad mitósica fue esporádica. Se realizaron reacciones de inmunohistoquímica para los marcadores señalados en la tabla 2. La única positividad útil para considerar la diferenciación posible fue para actina de músculo liso y, por lo tanto, se clasificó finalmente como 
Tabla 2. Pruebas de inmunohistoquímica realizadas y resultados

\begin{tabular}{|l|l|}
\hline Marcador & Resultado \\
\hline CD163 & Negativo \\
\hline CD68 & Negativo \\
\hline Calponina & Negativo \\
\hline Desmina & Negativo \\
\hline Mioglobina & Negativo \\
\hline Cromogranina & Negativo \\
\hline CD34 & Negativo \\
\hline Cocktail melanoma & Negativo \\
\hline Panqueratina & Negativo \\
\hline Proteína S100 & Negativo \\
\hline Actina específica de músculo liso & Positivo \\
\hline Ki67 & Negativo \\
\hline
\end{tabular}

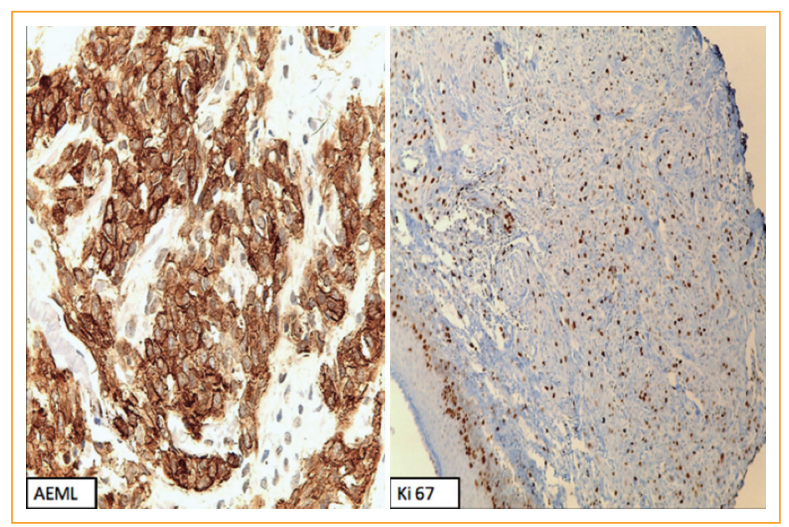

Figura 6. Estudio de inmunohistoquímica que reveló positivo para actina específica de músculo liso en toda la lesión, con un índice de proliferación Ki67 del 20\%.

leiomioma epitelioide con márgenes quirúrgicos libres (Fig. 6). Los marcadores negativos permitieron descartar las posibilidades de neoplasia histiocítica o de células de Langerhans, neoplasia neuroendocrina, de vaina nerviosa, epitelial o melanocítica.

\section{Discusión}

La primera condición para tratar cualquier tumor de los párpados es establecer un diagnóstico que obligatoriamente deberá ser corroborado por histopatología.
Los leiomiomas son neoplasias originarias del músculo liso. A nivel sistémico se encuentran principalmente en el útero. La localización intraocular y orbitaria es infrecuente. Se han reportado casos a nivel intraconal en un $46.2 \%$, extraconal en un $11.5 \%$ e intraextraconal en un $7.7 \%$ de los casos $^{5}$. Pueden alojarse en el compartimento anterior en el $30.8 \%$ o ser difusos en el $3.8 \%$ de los casos. El origen de estos tumores es incierto debido a la escasez de músculo liso en la órbita, solamente presente en los músculos cápsula-palpebral de Hessar y de Müller o en la pared de los vasos ${ }^{9,10}$.

Histológicamente, los leiomiomas presentan una población de células fusiformes con un patrón de distribución fascicular, citoplasma eosinófilo, retracción perinuclear y núcleos con bordes romos y sin actividad mitótica. En estudios inmunohistoquímicos, los leiomiomas son positivos para la actina del músculo liso, la desmina y la vimentina ${ }^{11}$. Los leiomiomas muestran un espectro que varía desde un escaso componente vascular a los hemangioleiomiomas, donde existe una profusa vascularización con vasos de calibre variable y gruesa capa muscular ${ }^{12}$. El diagnóstico diferencial incluye tumores benignos de células fusiformes, como el neurofibroma, el histiocitoma fibroso y el neurinoma. El leiomiosarcoma representa la forma maligna del leiomioma y muestra un alto grado de mitosis, atipia celular y necrosis ${ }^{13}$. La extirpación quirúrgica de la lesión y su biopsia es el tratamiento de elección.

\section{Conclusiones}

Las lesiones palpebrales tumorales y pseudotumorales son difíciles de diferenciar clínicamente, por lo que el abordaje y tratamiento siempre deberá ir acompañado de la toma de biopsia excisional y su análisis histopatológico.

\section{Conflicto de intereses}

Los autores declaran que no existe conflicto de intereses.

\section{Responsabilidades éticas}

Protección de personas y animales. Los autores declaran que para esta investigación no se han realizado experimentos en seres humanos ni en animales.

Confidencialidad de los datos. Los autores declaran que han seguido los protocolos de su centro de trabajo sobre la publicación de datos de pacientes. 
Derecho a la privacidad y consentimiento informado. Los autores han obtenido el consentimiento informado de los pacientes y/o sujetos referidos en el artículo. Este documento obra en poder del autor de correspondencia.

\section{Bibliografía}

1. Kanski JJ, Bowling B. Oftalmología clínica. 7. a ed. Elsevier; 2012. p. 4-5

2. Curutcher Mesner L, Peñate Santana H, Romero Báez S, Rutllán Civit J Medina Rivero F. Tumores palpebrales. Aspectos clínicos y su correlación con la histopatología de una series de 305 casos. Arch Soc Canar Oftal. 2012;23:40-5.

3. Audelan A. Tumor Palpebral. En:Enciclopedia práctica de medicina. Cap. V. 6- 0170/12.426.

4. Pe'er J. Pathoogy of eyelid tumors Indian. J Ophtalmol. 2016;64(3):177-90.
5. Hui JI, Buchser NM, Dubovy SR. Primary eyelid leiomyoma. Ophtalmic Plast Reconstr Surg. 2011;27(4):e102-3.

6. González-Martínez E, Robla Costales J, Fernández Fernández J, Viñuela Lobo J, Santamarta D, Lomas García J, et al. Leiomioma intraorbitario. Caso clínico y revisión de la literatura. Neurocirugia.2014;25(1):33-7.

7. Lin J, et al, Zhonghua Yan Ke Za Zhi, Clinicopathologic characteristics of angioleiomyoma of the eyelids and orbit. Zhonghua Yan Ke Za Zhi. 2015; 51(8):586-91.

8. Barsegian A, Ahmad A, Shinder R. Leiomyoma of the Palpebral Conjuntiva. Ophtalmology. 2017;124(1):65

9. Jakobiec FA, Jones IS, Tanenbaum M. Leiomyoma. An unusual tumour of the orbit. Br J Ophtalmol. 1973;57:825-31

10. Yan J, Zhou S, Li Y. Bening orbital tumors with bone destruction in children. PLoS One. 2012;7:e32111.

11. Gunduz K, Gunalp I, Erden E, Erekul S. Orbital leiomyoma: Report of a case and review of the literatura. Surv Ophtalmol. 2004;49:237-42.

12. Jakobiec FA, Jones IS, Tanenbaum M. Leiomyoma. An unusual tuour of the orbit. Br J Ophtalmol. 1973;57:825-31.

13. Wiechens B, Werner JA, Luttges J, Rudert $H$, Rochels R. Primary orbital leiomyoma and leiomyosarcoma. Ophthalmologica. 1999;213:159-64. 